\title{
Bilan de quinze années de surveillance hydrologique du site nucléaire de Cadarache (1962-ı 976)
}

\author{
H. ROUX, R. PAULIN, F. ROUX, J.-M. MORET et O. TARTARIN (*)
}

(Manuscrit reçu le 31 mai 1978)

\begin{abstract}
RÉSUMÉ
Le bilan relatif à 15 années de surveillance du site du C.E.N. de Cadarache permet d'individualiser, pour les eaux vives, trois périodes. La première période, de 1962 à 1963, qui correspond à la radioactivité spontanée avant tout rejet, met en évidence que : l'activité $\beta$ de la fraction soluble, élevée à cette époque en raison des retombées provoquées par les essais nucléaires en atmosphère libre (1961-1962), est directement liée à la pluviométrie; celle de la fraction insoluble est fonction de la teneur en matières en suspension, donc en relation avec le débit de la rivière; la radioactivité $\alpha$ est au contraire un fidèle reflet de la radioactivité naturelle. La deuxième période qui s'étend de 1964 à 1971 se caractérise par une diminution progressive de la moyenne annuelle de la radioactivité $\beta$ globale alors que le C.E.N. commençait dès décembre 1963, sous notre contrôle, ses rejets d'effluents liquides. La troisième période qui commence en 1972 se manifeste par un palier de radioactivité des eaux vives. En ce qui concerne les eaux potables, leur radioactivité n'a pas varié : elles sont restées insensibles aux retombées et aux rejets d'effluents.
\end{abstract}

\section{SUMMARY}

Evaluation of 15 years' surveillance of the C.E.N. site at Cadarache brings out three distinct periods as concerns the natural watercourses. A study of the first period, from 1962 to 1963, corresponding to spontaneous radioactivity before any release from the C.E.N., showed that $a$ ) Beta activity of the soluble fraction, very high then because of fall-out from atmospheric nuclear tests (1961-1962) was directly related to rainfall; the activity of the insoluble

(*) Avec la collaboration technique de M. Henri Bouteille.

Institut de recherches et d'applications médicales des isopotes radioactifs de l'Université d'Aix-Marseille-II, Faculté de Médecine, 27, boulevard Jean-Moulin, 13385 Marseille Cedex 4.

RADIOPROTECTION, VOL. $13-\mathrm{N}^{\circ} 3$ 
fraction was related to the amount of suspended matter and therefore depended directly on the mass flow of the river. b) Alpha radioactivity was, on the contrary, a faithful image of natural radioactivity. The second period, covering 1964-1971, was characterised by a progressive reduction of the annual average beta radioactivity although the C.E.N. started evacuating liquid effluents in December 1963. The third period, from 1972 on, is characterised by stable radioactivity of the watercourses. The radioactivity of drinking water has not varied at all, i. e. it has not been affected in any way by nuclear fall-out or by the release of liquid effluents.

\section{I. - INTRODUCTION}

Par protocole interministériel et en accord avec le Service Central de Protection contre les Radiations Ionisantes (S.C.P.R.I.), l'Institut de Recherches et d'Applications Médicales des Isotopes Radioactifs de l'Université d'AixMarseille II (I.R.A.M.I.R.) est chargé d'une mission de surveillance des rejets d'effluents radioactifs liquides du Centre d'Etudes Nucléaires de Cadarache (C.E.N.).

Cette mission, dont le bilan ci-après constitue le rapport de synthèse à moyen terme, a comporté deux étapes : la première correspond dès janvier 1962 , à l'établissement de la carte saisonnière de la radioactivité spontanée des eaux de la basse vallée de la Durance avant l'entrée en activité du C.E.N. Les premiers rejets effectués en décembre 1963 marquent le début de la deuxième étape qui se caractérise par la mise en œuvre de radioanalyses portant à la fois sur les effluents liquides avant rejet et sur les eaux du réceptable.

\section{II. - MÉTHODES}

\section{1) Protocole des PRÉLÈVements ( $f i g .1$ )}

Le C.E.N. de Cadarache est situé sur la rive gauche de la Durance, très légèrement en aval de la retenue de Jouques-Cadarache où s'achève la confluence de la Durance et du Verdon et où prend naissance le canal qui alimente en eau la ville de Marseille. Les rejets s'effectuent au droit du site nucléaire, donc en aval du barrage, dans les eaux résiduelles de la Durance qui rejoignent au sud d'Avignon le cours du Rhône.

De ce fait, s'il était utile pour l'étude du fond de radioactivité spontanée, avant donc que ne commencent les rejets d'effluents, de disposer, pour les eaux vives, de trois points de prélèvement en amont du C.E.N. :

point 1 : pont de Manosque $(-14 \mathrm{~km})$;

point 2 : pont de Vinon $(-6 \mathrm{~km})$;

point 3 : barrage de Cadarache $(-1 \mathrm{~km})$;

et de trois points en aval :

point 4 : pont de Mirabeau $(+4 \mathrm{~km})$;

point 5 : canal de Marseille $(+17 \mathrm{~km})$;

point 6 : pont de Pertuis $(+22 \mathrm{~km})$; 
on pouvait au contraire ne retenir, pour le contrôle hydrologique, depuis le début des rejets, que les points 3 et 4 qui rendent compte de manière significative de la radioactivité des eaux de la Durance en amont et en aval du point de rejet [1].

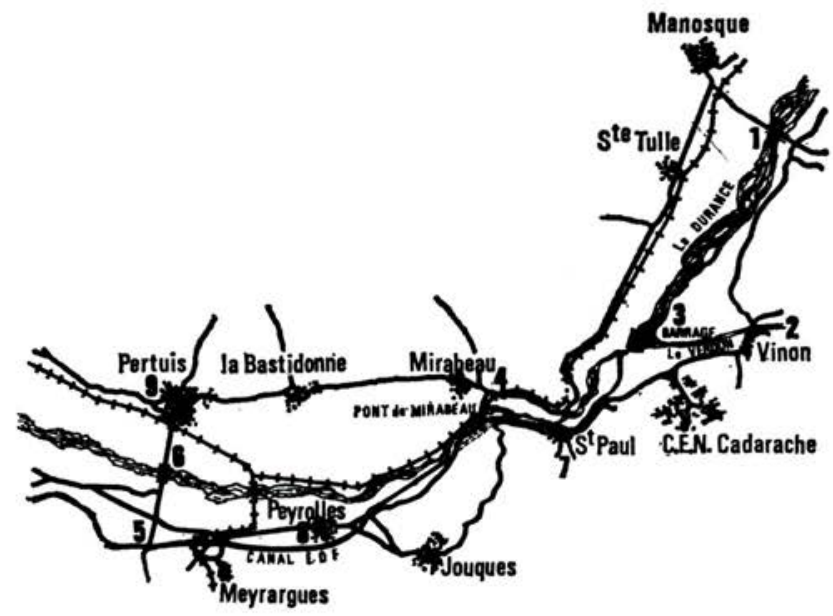

FIG. 1. - Points de prélèvements.

En ce qui concerne la surveillance des eaux potables, nous avons maintenu, au fil des années, les trois prélèvements suivants :

point 7 : Saint-Paul-lez-Durance (eau de source);

point 8 : Peyrolles (puits en berge filtrante);

point 9 : Pertuis (station de pompage puisant directement dans la nappe phréatique en bordure de Durance).

Nous avons effectué, pour un point donné, des prélèvements discontinus à un rythme hebdomadaire. La température de l'eau a été mesurée in situ et les conditions atmosphériques notées : précipitations, crues ou étiage, aspect boueux, etc. La mesure du pH de l'eau a été effectuée dès l'arrivée des échantillons au Laboratoire.

Pour les radioanalyses portant sur les effluents du C.E.N. de Cadarache, les prélèvements ont été pratiqués après homogénéisation, sur le contenu de chaque cuve de stockage d'effluents liquides en provenance des stations d'épuration et de décontamination. Compte tenu des résultats de ces radioanalyses, étaient fixées les conditions de dilution des rejets en Durance pour satisfaire aux normes eaux de boisson de la législation française.

\section{2) TeChNiques UtilisÉES POUR LE CONTRÔLE HYDROLOGIQUE}

La surveillance de la radioactivité des eaux vives et potables nécessitait de toute évidence la détermination des activités $\alpha$ et $\beta$ globales. Le radium 226 
étant le radionucléde de CMAP la plus sévère, il nous est apparu important de pouvoir l'identifier et le doser au même titre que l'uranium dont la présence est quasi inévitable dans les rejets d'un centre nucléaire.

a) Pour la détermination de l'activité $\alpha$ globale, nous avons adopté la méthode préconisée par FrançoIs [2]. Le résidu d'évaporation de 31 d'eau pour la fraction soluble et le résidu de calcination pour la fraction insoluble sont repris par l'acide nitrique. Une partie aliquote de la solution nitrique est déposée sur un disque scintillant de sulfure de zinc disposé sur la photocathode d'un photomultiplicateur.

b) Pour la mesure de la radioactivité $\beta$ globale, $200 \mathrm{mg}$ du résidu d'évaporation de 1 litre pour la fraction soluble et du résidu de calcination pour la fraction insoluble sont comptés avec un ensemble de détection $\beta$ à bas bruit de fond (ECF 103 INTERTECHNIQUE) à circulation d'un mélange hélium-isobutane, ayant un rendement de 0,3 pour le couple ${ }^{90} \mathrm{Sr}-{ }^{90} \mathrm{Y}$.

c) Le dosage de l'uranium a été pratiqué selon la méthode de PRICE [3] adaptée par CHARRIER [4] dont les principales étapes sont les suivantes : concentration des sels minéraux par évaporation de l'eau sur papier chromatographique, séparation de l'uranium par chromatographie ascendante, fusion par calcination avec du fluorure de sodium et comparaison fluorimétrique des intensités de fluorescence entre une solution étalon et l'eau à analyser. La limite de détection est de l'ordre de $0,1 \mu \mathrm{g} / 1$.

d) Le dosage du radium 226 a été effectué selon la méthode de l'émanation [5].

\section{3) RADiOANAlyse DES REJETS}

Dans un premier temps sont déterminées :

- l'activité $\alpha$ totale $(\alpha t)$ en utilisant un compteur proportionnel RA 15 INTERTECHNIQUE et un étalon de ${ }^{239} \mathrm{Pu}$;

- l'activité $\beta$ totale $(\beta t)$ par scintillation liquide [6];

- l'activité $\gamma$ totale avec un détecteur à scintillation à cristal de $\mathrm{NaI}$ en prenant comme étalon une source de ${ }^{137} \mathrm{Cs}$.

Ensuite sont effectués, pour chaque type de radioactivité $\alpha, \beta$ et $\gamma$, l'identification et le dosage de quelques radionucléides. Le choix de ces investigations particulières est dicté soit par l'abondance dans les effluents, soit par la radiotoxicité des émetteurs considérés, soit encore par l'amélioration technique des méthodes.

a) Pour ce qui est des radionucléides émetteurs $\alpha$, il convient de signaler que jusqu'en 1972 nous avons effectué régulièrement les dosages du ${ }^{226} \mathrm{Ra}$ et de l'uranium selon les techniques précitées. Par la suite, nous avons abandonné ces évaluations pour ne retenir que les déterminations de l'américium 241 et du plutonium 239 effectuées au moyen d'une chambre à grille INTERTECHNIQUE.

b) En ce qui concerne les émetteurs $\beta$, il est procédé à une évaluation globale de la radioactivité $\beta$ non molle $(\beta n m t)$ au moyen de l'E.C.F. 103, en prenant pour étalon le couple ${ }^{90} \mathrm{Sr}-{ }^{90} \mathrm{Y}$, ainsi qu'au dosage radiochimique spécifique du tritium et du couple ${ }^{90} \mathrm{Sr}-{ }^{90} \mathrm{Y}$ [7]. 
c) La spectrométrie $\gamma$, effectuée en utilisant un cristal-puits de cinq pouces et un analyseur 400 canaux INTERTECHNIQUE, équipé d'un correcteur de dérive, permet d'identifier essentiellement les pics d'activité $\gamma$ correspondant au couple ${ }^{106} \mathrm{Ru}-{ }^{106} \mathrm{Rh}$, au ${ }^{60} \mathrm{Co}$ et au ${ }^{137} \mathrm{Cs}$. L'activité de ce dernier radionucléide est généralement mesurable par spectrométrie quantitative. litre.

Tous les résultats de ces radioanalyses sont exprimés en picocuries par

\section{4) STrRatégie adoptéE POUR Les REJETS}

On sait [8] que si $n$ radionucléides sont rejetés, leur concentration respective $C_{1}, C_{2} \ldots C_{n}$ dans l'eau de rivière doit être telle que la somme des fractions correspondantes :

$$
\frac{C_{1}}{\mathrm{CMAP}_{1}}+\frac{C_{2}}{\mathrm{CMAP}_{2}}+\ldots+\frac{C_{n}}{\mathrm{CMAP}_{n}}
$$

soit inférieure ou égale à 1 .

C'est ce qu'exprime la formule classique de rejet :

$$
\sum_{p=1}^{n} \frac{C_{p}}{\mathrm{CMAP}_{p}} \leqq 1,
$$

où $C_{p}$ est la concentration du $p$-ième radionucléide et $\mathrm{CMAP}_{p}$ sa concentration maximale admissible (eau) à l'échelon population. D'après cette règle, il conviendrait que la composition qualitative et quantitative des effluents liquides soit parfaitement connue. Or, ceci est pratiquement impossible à réaliser. Aussi, avons-nous convenu d'une part de pousser le plus loin possible l'analyse de l'échantillon d'effluent de manière à établir avec le maximum de précision les premiers termes de la somme et d'autre part de calculer le dernier terme en prenant par sécurité pour valeur de la concentration $C_{n}$ la valeur de l'activité volumique correspondant aux radionucléides non identifiés et pour valeur de la $\mathrm{CMAP}_{n}$ celle du radioélément le plus radiotoxique non identifié pour le type de radioactivité considéré [9]. Illustrons l'application de la formule des rejets en nous plaçant dans le cas où la composition quantitative de l'effluent serait seule connue, soit par exemple : $90 \mathrm{pCi} / 1$ en activité $\alpha$ totale, $23000 \mathrm{pCi} / 1$ en activité $\beta$ totale et $9600 \mathrm{pCi} / 1$ en activité $\gamma$ totale d'énergie supérieure à $0,1 \mathrm{MeV}$. Nous devons alors attribuer l'activité $\alpha$ au ${ }^{226} \mathrm{Ra}$ de CMAP égale à $10 \mathrm{pCi} / 1$, l'activité $\beta$ totale au ${ }^{210} \mathrm{~Pb}$ de CMAP égale à $100 \mathrm{pCi} / 1$ dans le cas où le ${ }^{228} \mathrm{Ra}$ est absent de l'effluent et enfin l'activité $\gamma$ totale à ${ }^{126} \mathrm{I}$ de CMAP égale à $1000 \mathrm{pCi} / 1$. La formule des rejets impose une dilution « $d$ » telle que le produit :

$$
d\left(\frac{90}{10}+\frac{23000}{100}+\frac{9600}{1000}\right)
$$

soit au plus égal à 1 ce qui conduit à une valeur de $d$ pratiquement égale à $1 / 250$.

voL. $13-\mathrm{N}^{\circ} 3$ 
a) Dans le cas de la radioactivité $\alpha$, nous avons adopté successivement deux modes d'évaluation de la concentration $C_{n \alpha}$ des radionucléides des émetteurs $\alpha$ non identifiés.

Jusqu'en 1972, le calcul de $C_{n \alpha}$ s'effectuait en opérant la différence entre l'activité volumique alpha totale $(\alpha t)$ et les activités volumiques correspondant au radium 226 et à l'uranium :

$$
C_{n \alpha}=(\alpha t)-\left({ }^{226} \mathrm{Ra}+\mathrm{U}\right)
$$

Cette radioactivité $\alpha$ non identifiée était attribuée au ${ }^{223} \mathrm{Ra}$ dont la CMAP est de $700 \mathrm{pCi} / 1$.Ce mode d'estimation permettait de reconnaître dans l'activité volumique alpha totale la part prise par le ${ }^{226} \mathrm{Ra}$ dont la CMAP de $10 \mathrm{pCi} / 1$ est la plus sévère de toutes les CMAP. Nous déterminons ainsi la contribution à la dilution finale imposée par l'élément de CMAP la plus faible et donc la plus contraignante.

Toutefois, l'expérience nous a montré que la méthode de dosage du ${ }^{226} \mathrm{Ra}$ par la méthode de l'émanation ne fournissait que des valeurs par défaut. Aussi, avons-nous estimé, à partir de 1973, qu'il était plus sûr, pour calculer $C_{n \alpha}$, d'effectuer la différence entre l'activité volumique $\alpha$ totale et les activités volumiques correspondant à ${ }^{241} \mathrm{Am}$ et ${ }^{239} \mathrm{Pu}$ :

$$
C_{n \alpha}=(\alpha t)-\left({ }^{241} \mathrm{Am}+{ }^{239} \mathrm{Pu}\right)
$$

La radioactivité $\alpha$ non identifiée est, dès lors, en l'absence de son dosage, attribuée entièrement au ${ }^{226} \mathrm{Ra}$. Comme cette $C_{n \alpha}$, calculée selon l'expression (2) est nettement supérieure à l'activité volumique obtenue antérieurement avec le dosage du radium 226, il s'ensuit une augmentation importante de la marge de sécurité.

b) Pour la radioactivité $\beta$ molle, la concentration $C_{n \beta m}$ des émetteurs $\beta$ de faible énergie non identifiés résulte de la soustraction des activités volumiques respectives du tritium $\left({ }^{3} \mathrm{H}\right)$ et des bétas mous totaux $(\beta m t)$ :

$$
C_{n \beta m}=(\beta m t)-\left({ }^{3} \mathrm{H}\right) ;
$$

avec

$$
(\beta m t)=(\beta t)-(\beta n m t) .
$$

La CMAP adoptée dans ce dernier cas est celle du ${ }^{228} \mathrm{Ra}$ égale à $30 \mathrm{pCi} / 1$ : ceci bien que $1 \mathrm{e}^{232} \mathrm{Th}$ ne subisse au C.E.N. de Cadarache aucune transformation.

c) En ce qui concerne la radioactivité des émetteurs $\beta$ d'énergie moyenne et élevée, il convient pour établir la concentration $C_{n \beta n m}$ de l'activité $\beta$ non molle de calculer la différence entre la radioactivité $\beta$ non molle globale et l'ensemble représenté par les concentration d'émetteurs $\beta \gamma$ identifiés :

$$
C_{n \beta n m}=(\beta n m t)-\left({ }^{131} \mathrm{I}+{ }^{60} \mathrm{Co}+{ }^{137} \mathrm{Cs}+{ }^{106} \mathrm{Ru} \cdot{ }^{106} \mathrm{Rh}+{ }^{90} \mathrm{Sr} .{ }^{90} \mathrm{Y}\right) .
$$

Dans ce cas, la CMAP retenue est celle de l'iode 126 qui est égale à $1000 \mathrm{pCi} / 1$. 
Lors des premiers rejets, nous ne prenions pas en compte l'iode $126 \mathrm{du}$ fait que le pic gamma de $0,39 \mathrm{MeV}$ était toujours absent du spectre. Mais il nous a semblé préférable, depuis les cinq dernières années, de rendre l'iode 126 responsable de l'activité non identifiée $\beta$ non molle en raison de la difficulté que l'on peut avoir à différencier le pic de l'iode 126 de celui de l'iode 131.

\section{III. - RÉSULTATS}

\section{1) SuRVeillance hydrologique}

Nous ne pouvons pas, en raison du volume important d'informations, faire figurer tous les résultats hebdomadaires accumulés en 15 années et qui ont été publiés par ailleurs $[1,10-13,15]$.

TABLEAU

MOYenNes anNuelles de LA RAdioactivité $\alpha$ et $\beta$ (en pCi/l)

\begin{tabular}{|c|c|c|c|c|c|c|c|c|c|c|}
\hline & \multicolumn{4}{|c|}{ Eaux vives } & \multicolumn{6}{|c|}{ Eaux potables } \\
\hline & \multicolumn{2}{|c|}{$\begin{array}{c}\text { Amont } \\
\text { de } \\
\text { Cadarache }\end{array}$} & \multicolumn{2}{|c|}{$\begin{array}{c}\text { Aval } \\
\text { de } \\
\text { Cadarache }\end{array}$} & \multicolumn{2}{|c|}{ Saint-Paul } & \multicolumn{2}{|c|}{ Pertuis } & \multicolumn{2}{|c|}{ Peyrolles } \\
\hline & $\alpha$ & $\beta$ & $\alpha$ & $\beta$ & $\alpha$ & $\beta$ & $\alpha$ & $\beta$ & $\alpha$ & $\beta$ \\
\hline $1962 \ldots$ & 5,4 & 26,8 & 5,7 & 11,1 & 4,7 & 5,6 & 3,9 & 4,1 & 6,3 & 5,6 \\
\hline $1963 \ldots$ & 5,2 & 46 & 3 & 42 & 2,1 & 3 & 2,2 & 5,8 & 3,9 & 6 \\
\hline $1964 \ldots$ & 5,9 & 8,4 & 6,4 & 7 & 5 & 1,6 & 4,7 & 3,4 & 4,7 & 2,5 \\
\hline $1965 \ldots$ & 7,5 & 8 & 7 & 9,3 & 7,4 & 2,5 & 7,6 & 6,3 & 8,3 & 4,2 \\
\hline $1966 \ldots$ & 9,9 & 5,4 & 8,2 & 5,7 & 9,6 & 3,9 & 9,6 & 5,1 & 9,1 & 4,6 \\
\hline $1967 \ldots$ & 5,7 & 3,5 & 5 & 3,8 & 4,5 & 2,3 & 5,8 & 4,2 & 4,9 & 3 \\
\hline $1968 \ldots$ & 4,9 & 3 & 3,1 & 3,2 & 2,4 & 2,5 & 4,3 & 2,2 & 2,2 & 3,3 \\
\hline $1969 \ldots$ & 7,6 & 7,1 & 7,8 & 6,2 & 6,1 & 3,8 & 4,6 & 5,8 & 5,5 & 4 \\
\hline $1970 \ldots$ & 6,7 & 5,2 & 6,7 & 4,4 & 3,6 & 3 & 4,9 & 4,9 & 4 & 3,3 \\
\hline $1971 \ldots$ & 4,2 & 4,3 & 3,4 & 3,1 & 2,2 & 2,6 & 2 & 4 & 2 & 3,1 \\
\hline $1972 \ldots$ & 3,9 & 3,9 & 3 & 2,7 & 1,7 & 2,9 & 2 & 4,5 & 2,5 & 3 \\
\hline $1973 \ldots$ & 3,5 & 3,4 & 2,4 & 3,4 & 1,7 & 2,3 & 1,9 & 2,6 & 1,9 & 2,6 \\
\hline $1974 \ldots$ & 3,6 & 2,9 & 3 & 2,1 & 1,6 & 2 & 1,6 & 5,2 & 1,4 & 2,6 \\
\hline $1975 \ldots$ & 4,7 & 5 & 4 & 4,5 & 3,3 & 3,1 & 3,7 & 5,4 & 4 & 4,4 \\
\hline $1976 \ldots$ & 1,5 & 6 & 1,1 & 3,6 & $<1$ & 2,3 & 1,4 & 5,2 & 1 & 2,8 \\
\hline
\end{tabular}




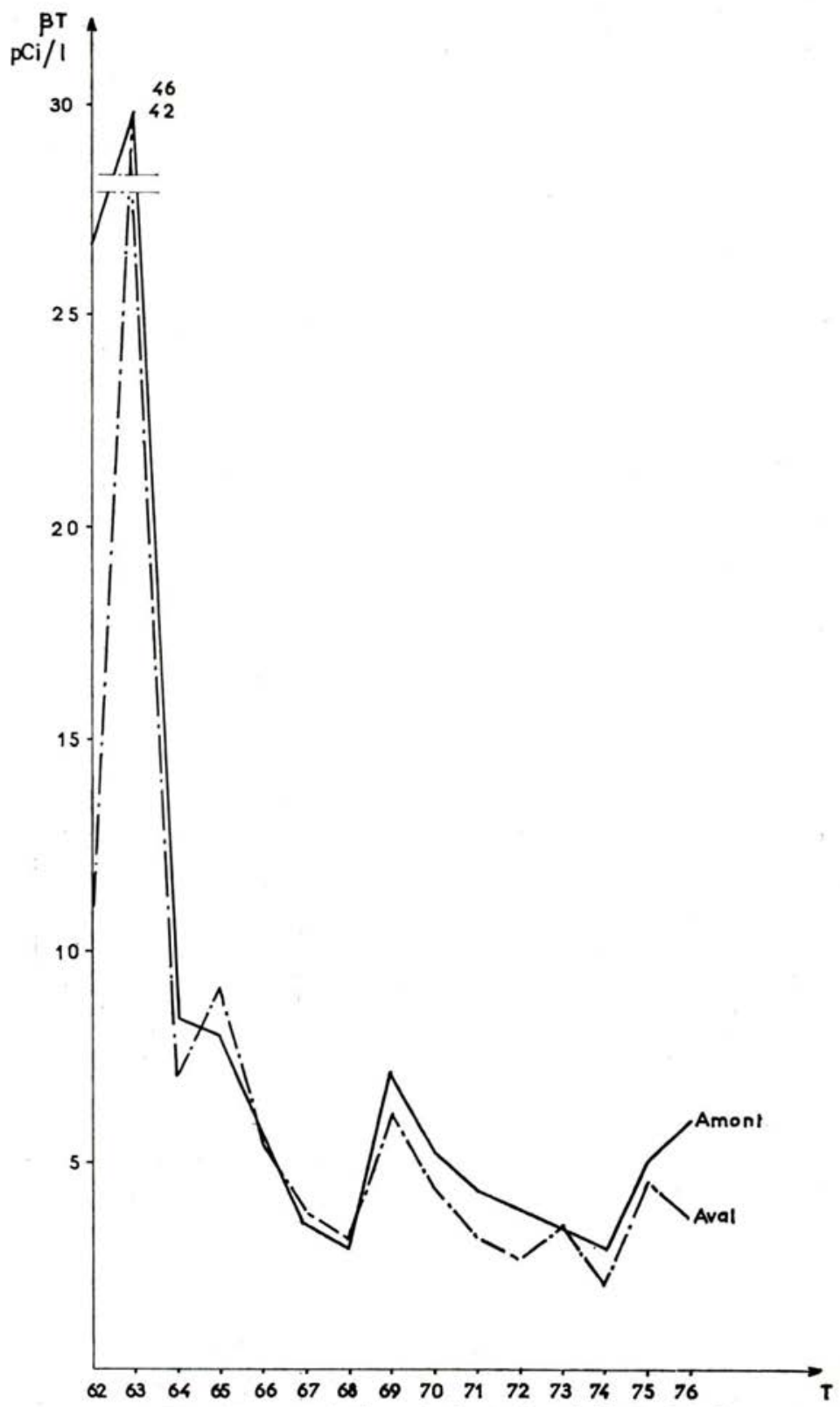

FIG. 2. - Variations de la radioactivité $\beta$ globale

dans les eaux de la Durance en amont et en aval de Cadarache. 
Nous faisons seulement figurer dans le tableau les moyennes annuelles des radioactivités $\alpha$ et $\beta$ globales pour les années 1962 à 1976. Pour alléger la lecture du tableau, nous ne mentionnerons pas les teneurs en radium 226 et en uranium dont les valeurs basses ont toujours été à la limite de la sensibilité des méthodes. Bien que ces valeurs n'aient pas été portées au tableau, précisons bien que l'estimation du radium 226 a toujours été pratiquée par la méthode de l'émanation jusqu'en 1973 et depuis 1973 par la technique indiquée ci-dessus (II-4-a).

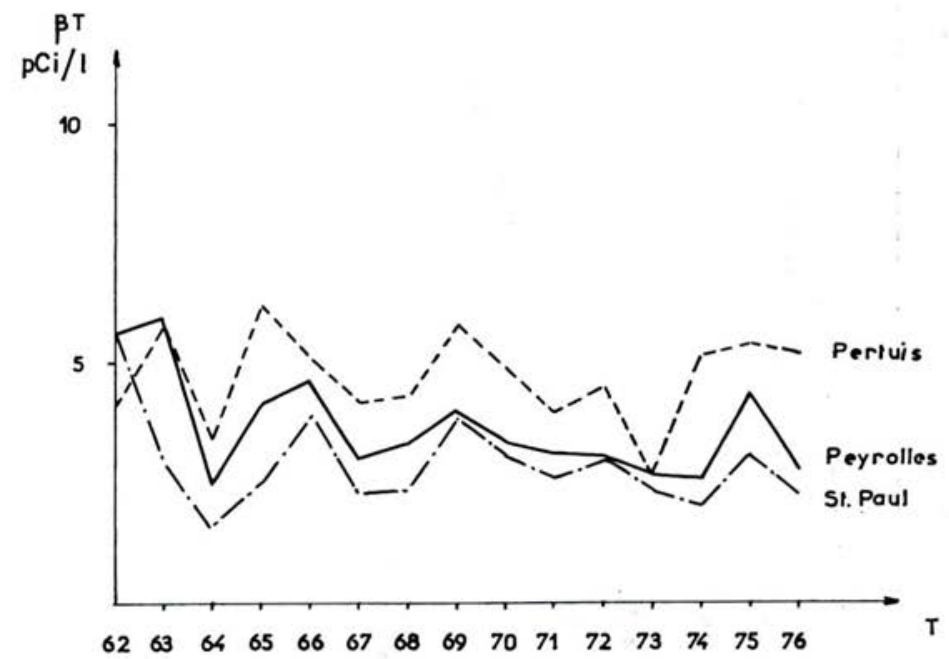

FIG. 3. - Évolution de la radioactivité $\beta$ de la fraction soluble des eaux potables de Saint-Paul-lez-Durance, de Peyrolles et de Pertuis.

La figure 2 rend compte de la variation en fonction du temps de la radioactivité $\beta$ globale pour les deux principaux points de prélèvement en eaux vives en amont et en aval du point de rejet, c'est-à-dire pour les points 3 et 4. La figure 3 traduit l'évolution du même type de radioactivité pour les prélèvements d'eaux potables.

\section{2) ANALyse des efFluents}

$\mathrm{Si}$, pour des raisons déontologiques, il ne nous est pas possible de donner le résultat détaillé des radioanalyses des effluents, nous ferons remarquer que, pratiquement, la quasi-totalité de l'activité $\alpha$ est supportée par l'américium 241 et le plutonium 239 et que la majeure partie de l'activité $\beta$ non molle est supportée par le couple ${ }^{90} \mathrm{Sr}-{ }^{90} \mathrm{Y}$ et le ${ }^{137} \mathrm{Cs}$ qui représente, par ailleurs, la plus grande partie de l'activité $\gamma$.

En limitant, dès lors, nos réflexions aux deux paramètres $(\alpha t)$ et $(\beta n m t)$ nous avons remarqué ( fig. 4) que le maximum pour ( $\alpha t$ ) a été atteint en 1964 et pour $(\beta n m t)$ au premier semestre 1971. En fonction de ces résultats nous avons établi un index annuel de concentration Ic des effluents liquides. Cet 
index est calculé en faisant le rapport entre la concentration moyenne annuelle et la valeur du maximum précisé ci-dessus. Les variations des valeurs de Ic pour la radioactivité $\alpha$ totale et pour la radioactivité $\beta$ non molle sont mises en évidence sur la figure 4.

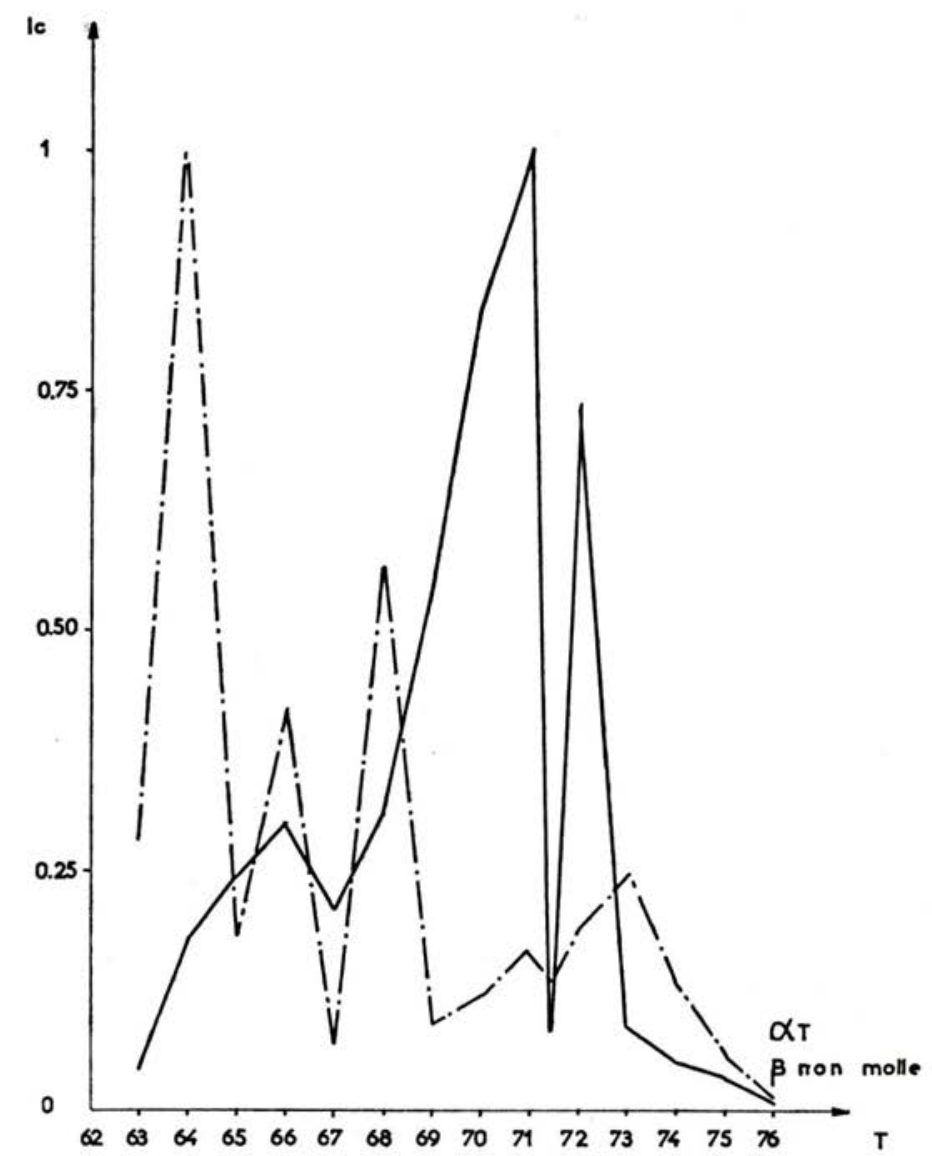

FIG. 4. - Variations de l'index de concentration (Ic) des effluents liquides du C.E.N. de Cadarache (activité $\alpha$ totale et l'activité $\beta$ non molle).

\section{IV. - DISCUSSION}

Nous pouvons diviser la période qui s'étend de janvier 1962 à décembre 1976 en trois tranches de temps.

a) La première tranche se rapporte aux années 1962 et 1963 pendant lesquelles le problème des rejets du C.E.N. de Cadarache, alors en construction, 
ne se posait pas. Or, les valeurs de la radioactivité $\beta$ des eaux vives sont les plus élevées que nous ayons enregistrées, puisque les moyennes annuelles se situaient en aval de Cadarache respectivement à 11 et $42 \mathrm{pCi} / 1$ au cours des années 1962 et 1963 . Ces valeurs, comme l'a montré l'un de nous [1] doivent être reliées aux retombées radioactives provoquées par les explosions nucléaires expérimentales en atmosphère libre des années 1961 et 1962 . Ce fait est confirmé par les valeurs plus élevées enregistrées au cours des mois de grande pluviométrie ( fig. 5). L'examen de ce graphique met nettement en évidence les

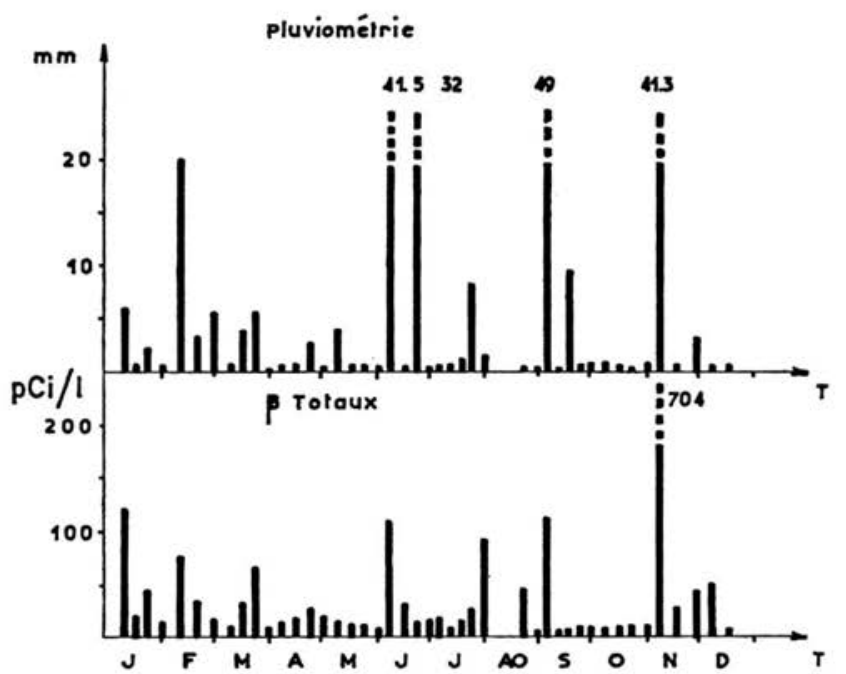

Fig. 5. - Corrélations entre la pluviométrie et la radioactivité $\beta$ globale des eaux de la Durance prélevées au pont de Mirabeau (année 1963).

corrélations qui existent entre la radioactivité $\beta$ d'une part et les conditions atmosphériques d'autre part. On voit qu'une augmentation de la pluviométrie se traduit, presque dans tous les cas, par une élévation de l'activité $\beta$ globale. En particulier, une pluviométrie moyenne, après une longue période de sécheresse, peut entraîner des activités $\beta$ élevées. Cettec orrélation est moins évidente pour l'activité $\alpha$, mais peut exister.

De plus, confirmant notre hypothèse, les valeurs de radioactivité $\beta$ enregistrées en amont du site étaient supérieures à celles trouvées en aval : $27 \mathrm{pCi} / 1$ en 1962 et 46 pCi/l en 1963. Il convient également de signaler qu'il en sera pratiquement toujours ainsi au cours des années comme le montre la figure 2 .

b) La deuxième période qui s'étend de décembre 1963 à juillet 1971 se caractérise par une diminution progressive de la moyenne annuelle de la radioactivité $\beta$ globale qui passe de $9 \mathrm{pCi} / 1$ en 1964 à $4 \mathrm{pCi} / 1$ au premier semestre 1971 alors que le C.E.N. commençait dès le mois de décembre 1963 et poursuivait sous notre contrôle ses rejets. Seule, la radioactivité $\alpha$ s'élève très légèrement en 1965 et 1966 pour retomber dès 1971 aux valeurs de base 
des années 1962 et 1963. Soulignons à nouveau que des valeurs du même ordre sont trouvées tant en amont qu'en aval, ce qui exclut encore la mise en cause du Centre.

Certes, nous imposions au Centre des normes sévères de rejet, d'autant plus que, comme le montre la figure 4, l'activité des effluents au cours de cette époque était relativement élevée : les index de concentration étant la plupart du temps compris entre 0,5 et 1 . Néanmoins, nous pensons que la baisse de la radioactivité $\beta$ des eaux vives de la Durance doit en priorité être imputée à l'arrêt des explosions nucléaires en atmosphère libre.

Notre action de protection par sa rigueur ne permet pas d'enregistrer, durant cette période, une élévation sensible de la radioactivité naturelle.

c) La troisième époque qui commence au deuxième semestre de 1971 pour s'achever en décembre 1976 se manifeste par un palier de concentration légèrement descendant oscillant autour de $4 \mathrm{pCi} / \mathrm{l}$ suivi d'une très légère augmentation en 1975. Cette variation se retrouve tant au niveau de la radioactivité $\alpha$ que $\beta$ et tant en aval qu'en amont.

Le plateau actuel coïncide avec la mise en route par le C.E.N. au cours du deuxième semestre de 1971 d'un système de décontamination par distillation. C'est donc le distillat faiblement radioactif qui constitue désormais l'effluent liquide destiné au rejet en Durance. La figure 4 démontre l'efficacité de ce dispositif puisque les index de concentration $\beta$ enregistrés au cours des trois dernières années sont toujours inférieurs à 0,1 . La radioactivité $\beta$ des effluents a ainsi diminué en moyenne d'un facteur 10 .

En fait, comme les valeurs trouvées en amont et en aval du Centre sont du même ordre de grandeur, l'influence du C.E.N. doit à nouveau être écartée.

La cause de la légère augmentation enregistrée en amont et en aval en 1975 et en amont en 1976 peut être imputée à la pluviométrie élevée enregistrée dans cette région au cours de ces deux dernières années. Mais s'il n'est pas possible d'apporter une explication formelle, il convient de remarquer que cette légère remontée correspond à une augmentation des retombées $\beta$ à vie longue enregistrée au cours du premier semestre 1975. Cette augmentation est également apparente quand on consulte les résultats obtenus pour les eaux de surface, pendant cette période, par le National Institute of Radiological Sciences de Chiba au Japon et le Department of Environmental Conservation de l'État de New York.

En ce qui concerne les eaux potables, leur radioactivité n'a pas varié ( fig. 3) : elles sont restées insensibles aux retombées et aux rejets.

Par ailleurs, si on compare la variation de la radioactivité $\beta$ des eaux vives ( fig. 2) avec l'évolution, pour la même période, des doses d'irradiation externe artificielle annuelles calculées par Watanabe [14], on constate une similitude qui confirme le rôle primordial des retombées dans la radioactivité des eaux de surface et minimise celui des rejets d'effluents. En effet, selon cet auteur, l'irradiation externe annuelle moyenne de l'homme est passée de 40 mrem en 1962 à 1,8 mrem en 1974. 
En conclusion, cette étude, à moyen terme, rend compte de l'efficacité des dispositifs de radioprotection adoptés et de l'absence de retentissement des rejets du C.E.N. de Cadarache sur l'environnement.

\section{BIBLIOGRAPHIE}

[1] Paulin R. Étude de la radioactivité spontanée des eaux de la basse Durance. Thèse doctorat d'État en Pharmacie, Marseille, 1966.

[2] Francois H. Analyse et contrôle de protection. Bull. Inf. Sc. Techn. C.E.A., 1962, 58, 50-52.

[3] Price G. R., Feretti R. J., Schwartz A. Fluoro-photometric determination of uranium. Analyt. Chem., 1953, 25, 322-325.

[4] Charrier J. P. Application de la méthode fluorimétrique de dosage de l'uranium à l'étude de la radioactivité spontanée des eaux de la Durance. Mémoire Méd. d'or. Intern. Pharmacie, Marseille, 1963.

[5] JACOBI R. B. The determination of radon and radium in water. J. Chem. Soc., Suppl., 1949, 314-319.

[6] Briand C., Roux H. Étude d'un mélange de radioéléments émetteurs $\beta$. Radioprotection, 1967, 2, 179-187.

[7] Briand C., Monges A. M., Regis L. Dosage du ${ }^{90} \mathrm{Sr}$ et du ${ }^{89} \mathrm{Sr}$ dans le cadre de la surveillance d'un site nucléaire. Bull. Soc. Chim. France., 1969, 14, 28.

[8] BRIAND C. Quelques aspects du problème posé par le rejet des effluents radioactifs du C.E.N. de Cadarache dans la Durance. Bull. Soc. Pharm., Marseille, 1967, 16, 230-236.

[9] Roux H. Contrôle du rejet des effluents radioactifs liquides du C.E.N. de Cadarache. Rapport IRAMIR $\mathrm{n}^{\circ}$ 5, 1966.

[10] Roux H., Crevat A., Paulin R., Bouteille H. Radioactivité des eaux de la Durance. Rapport aux Nations Unies, New York, 1963.

[11] Crevat A., Paulin R., Bouteille H. Mesure de la radioactivité spontanée des eaux de la Durance, 1962-1963. Pharmacie Médit., 1964, 5, 556-572.

[12] Briand C., Paulin R. Bilan de cinq années de contrôle de la radioactivité des eaux de la Durance. Bull. Soc. Pharm., Marseille, 1967, 16, 238-244.

[13] Roux H. Résultats des mesures de la radioactivité des eaux de la Durance. Rapports IRAMIR, $\mathrm{n}^{\circ} 1$ à 7.

[14] Watanabe H. In Radioactivity survey data in Japan, Rapport japonais NIRS-RSD, $\mathrm{n}^{\circ} 40,1975,21-22$.

[15] Roux F., Chauvet M., Bisset J.-P. Surveillance hydrologique du site du C.E.N. de Cadarache. $7^{\circ}$ Congrès national A.T.S.R., Marseille, mai 1972, 47-51. 